\title{
Semi-automated color segmentation of anatomical tissue
}

\author{
C. Imelińska ${ }^{\mathrm{a}, *}$, M.S. Downes ${ }^{\mathrm{b}}$, W. Yuan ${ }^{\mathrm{c}}$ \\ ${ }^{\mathrm{a}}$ Department of Computer Science, Columbia University and Office of Scholarly Resources, College of Physicians and Surgeons of Columbia University, \\ 701 W. 168th St., HHHSC 201, New York, NY 10032, USA \\ ${ }^{\mathrm{b}}$ Department of Electrical Engineering and Computer Science, University of California at Berkeley, Soda Hall, Berkeley, CA 94720, USA \\ ${ }^{\mathrm{c}}$ Department of Computer Science, Stanford University, Gates Computer Science Building, Stanford, CA 94305, USA
}

Received 6 December 1999

\begin{abstract}
We propose a semi-automated region-based color segmentation algorithm to extract anatomical structures, including soft tissues, in the color anatomy slices of the Visible Human data. Our approach is based on repeatedly dividing an image into regions using Voronoi diagrams and classifying the regions based on experimental classification statistics. The user has the option of reclassifying regions in order to improve the final boundary. Our results indicate that the algorithm can find accurate outlines in a small number of iterations and that manual interaction can markedly improve the outline. This approach can be extended to 3D color segmentation. (C) 2000 Published by Elsevier Science Ltd. All rights reserved.
\end{abstract}

Keywords: Image processing; Medical visualizations; Computational geometry; Computer graphics

\section{Introduction}

Over the last decade, rapid advances in computer graphics hardware and software have spawned a variety of new fields. Medical informatics has quickly become one of the most active of these pursuits. Research in medical informatics hinges primarily on integrating computer technology into the practice of medicine to improve all areas of the field from education to diagnosis and treatment. In particular, many applications in the field involve the visualization and manipulation of medical image data, such as MRI, $\mathrm{CT}$, and PET scans, which require sophisticated techniques from computer graphics and other disciplines. In an effort to advance the state of the art in medical imaging and to improve upon the tools currently available for medical education and diagnosis, the National Library of Medicine initiated the Visible Human Project [1,2].

Participants in the project are working toward producing a complete library of high-resolution color 3D representations of an adult male and a female cadaver. The initial phase of the project, carried out by the University of Colorado, involved the generation of true-color 2D slides from cryosections of the male and female cadavers [2]. The slides

\footnotetext{
* Corresponding author. Tel.: + 1-212-305-1990; fax: + 1-212-3058388.

E-mail address: ci42@columbia.edu (C. Imelińska).
}

allow for the representation of details which had been all but invisible in more traditional data sets. However, in order to make the best use of the data, an accurate and efficient method must be developed to identify structures within the individual 2D slices. Outlines of the structures can then be used to extract 3D voxel-based models and 3D surface-defined wrappings from the 2D data, see Fig. 1. These models must not only be accurate enough to allow for labeling of all important anatomical structures, but must also be space efficient enough to be usable in interactive applications, such as the The Vesalius ${ }^{\mathrm{rt}}$ Project, ${ }^{1}$ an interdisciplinary effort at Columbia University to create a network-based, platform-independent electronic "course" in anatomy [3-9].

Generating outlines for the structures, which falls under the umbrella of image segmentation, stands out as one of the most challenging and vital phases of the project, primarily because the results of all subsequent steps in the process depend on the quality of the initial 2D segmentation. For many years, work in the field centered on segmenting gray scale images, due primarily to the fact that, until recently, computer systems were not powerful enough to display and manipulate large, full-color data sets. Research focused primarily on two different approaches to the segmentation problem: region-based and edge-based methods. Regionbased methods take the basic approach of dividing the image into regions and classifying pixels as inside, outside, 

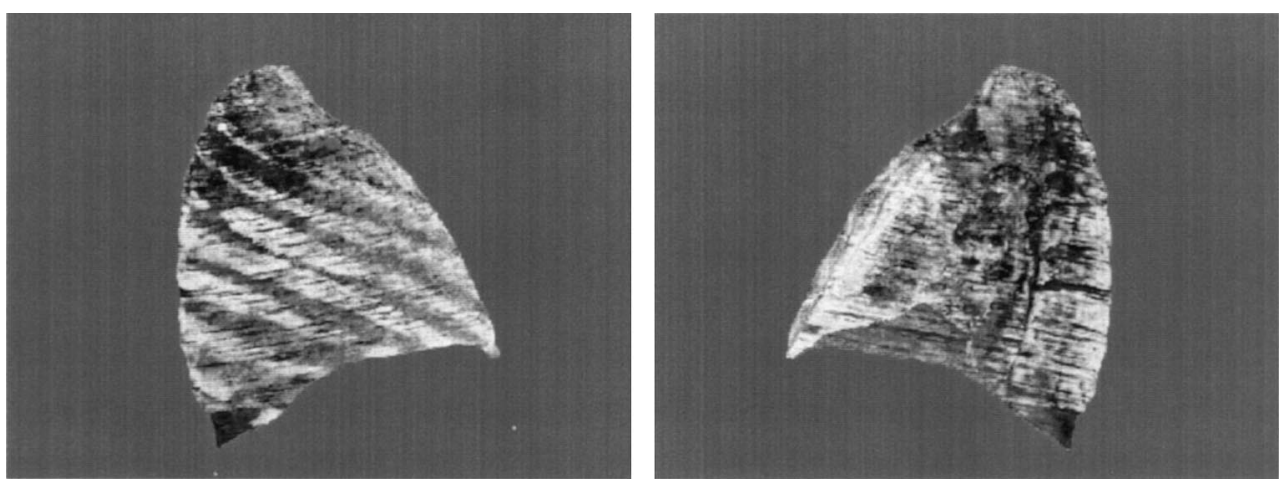

Fig. 1. Vesalius ${ }^{\mathrm{TN}}$ project-right lung.

or on the boundary of a structure based on its location and the surrounding $2 \mathrm{D}$ regions. On the other hand, the edge-based approach classifies pixels using a numerical test for a property such as image gradient or curvature [10]. In particular, there are model-based techniques like snakes [11] which start with an initial deformable boundary curve and try to align this estimated boundary with the actual boundary of the region of interest using gradient features. Recently, hybrid methods combining the model-based and region-based techniques have been considered [12].

The advent of more powerful and easily accessible hardware brought about a shift in the flow of research toward the more widely applicable and more complex problem of color segmentation. Indeed, the field has finally begun to witness the publication of a sizable body of research in the area of color image segmentation as opposed to gray scale [13-15]. Much of the work currently being pursued involves the extension of various gray scale methods to the realm of color images. These efforts represent a challenging advance considering that working with color images requires one to address a variety of issues, such as conversion between different color spaces and manipulation of larger volumes of data as opposed to gray scale. The introduction of the Visible Human data sets by the National Library of Medicine provides an opportunity to test new color segmentation methods against the most detailed and complete color anatomical images ever created.

Along this line, we propose a new method of 2D color image segmentation which builds upon previous work in gray scale segmentation. Bertin and Chassery have presented a gray scale region-based segmentation method for microscopic data which makes use of Voronoi diagrams to divide the image into regions [16]. In 2D, a Voronoi diagram is a structure which divides the Euclidean plane, for a given set of input seed points, into regions called Voronoi regions each of which contains all the points closer to its seed point than any other seed point [17]. The gray scale method involves distributing seed points in a microscopy data set and generating a Voronoi diagram for these seeds. Each Voronoi region is visited in order to determine if it is homogeneous, either inside or outside of the structure, or heterogeneous, i.e. on the boundary of the structure of interest. The boundary regions are further subdivided by adding more seed points and the classification is repeated until all regions are found to be homogeneous.

In extending this approach to color anatomical images, we have addressed a variety of new problems. For example, in a typical slide from the Visible Human data, it is possible to define a set of classification statistics which describes a particular anatomical structure. However, the rest of the image is filled with various other anatomical structures that form an extremely heterogeneous environment. This heterogeneity outside the region of interest prevents us from identifying homogeneous exterior regions and leads us to an algorithm based on identifying interior and noninterior regions, as described below. In addition, we provide the user with the option of interactively reclassifying regions while the algorithm iterates. As will be shown, a minimal amount of interaction markedly improves the segmentation results. Rather than terminating when all regions are homogeneous, our algorithm runs for a userspecified number of iterations. Also, we are using a more accurate and efficient algorithm for the classification of Voronoi regions.

The existing segmentation algorithms used in most applications for the Visible Human data can allow for a good quality rendering of skin, bones, and muscles, but not for most of the "soft-tissue" organs like the heart, bronchial tree or lungs. Unless a thorough color and texture classification of different types of human tissues is created, complete processing of new cadavers will have to be done manually. We have developed a color segmentation algorithm for the lung extraction as a first attempt to build a collection of similar tools for the other organs. When applied to the Visible Human Project cryosection slides, our semi-automated system produces accurate outlines for the lungs, which have in the past presented difficulties for more traditional approaches. 


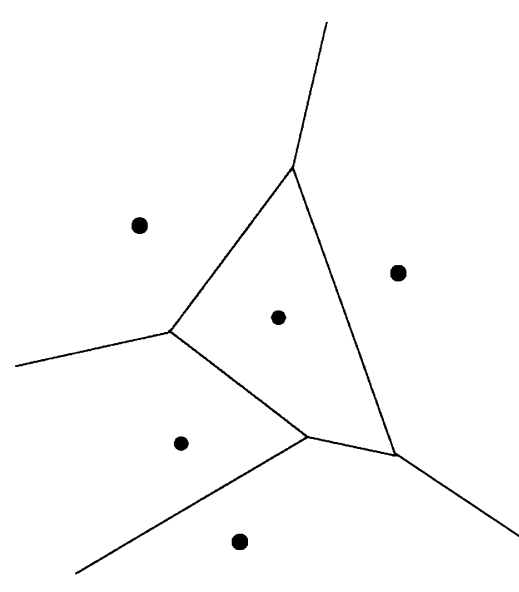

(a)

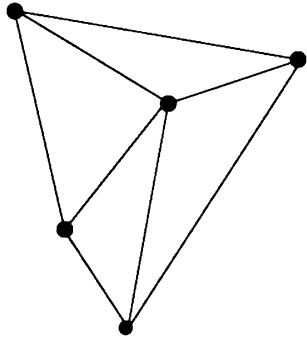

(b)

Fig. 2. (a) Voronoi diagram. (b) Delaunay triangulation.

\section{Region-based segmentation algorithm}

In approaching the problem of segmenting color anatomical data, we recognized a need for a new method to supplement the traditional techniques used in segmentation. We propose a region-based algorithm which quickly converges to an accurate boundary and requires minimal user interaction. Our basic approach is to subdivide an image into regions, classify each region as either inside or outside the target structure, and then break up the regions on the boundary between the two classifications into smaller regions and repeat the classification and subdivision on the new set of regions. This process can be repeated as many times as the user wishes, within the bounds of hardware limitations, in order to refine the calculated boundary.

Voronoi diagram and Delaunay triangulation play a central role in the algorithm. In 2D the Voronoi diagram for a set of $|V|$ seed points is a partition of the Euclidean plane into Voronoi regions of points closer to one point of $V$ than to any others, see Fig. 2(a). The Voronoi region of a point $v \in V$ is a set of points in the plane closer to $v$ than to

\section{Input set of $\mathrm{n}$ points.}

2. Compute Voronoi Diagram.

3. For each Voronoi region, classify it as interior or exterior.

4. Label a subset of the exterior regions as boundary regions.

5. (Optional:) Manually reclassify regions.

6. Compute Delaunay triangulation and display segments which connect boundary regions.

7. Add seeds to the edges of boundary regions.

8. Goto 2 until specified number of iteration are completed or until user chooses to quit.

Fig. 3. Pseudocode for the 2D region-based color segmentation algorithm. any other point $u \in V, v \neq u$, [17]. Two Voronoi regions are adjacent if they share a Voronoi edge. The Delaunay triangulation of $V$ can be derived from the corresponding Voronoi diagram by joining each of the two points whose Voronoi regions are adjacent, see Fig. 2(b).

We divide an image, using the Voronoi diagram, into regions by distributing, either manually or automatically, a number of seed points throughout the plane of the image and then generating the Voronoi diagram of these points. (We will show later that the automatic point distribution is sufficient.) Each Voronoi region is a convex polygon that can be efficiently analyzed for various statistics, including the mean color intensities and their variances. After collecting data for a region, we can classify it as interior or exterior with respect to the structure of interest. Of course, the classification criteria vary according to the type of image being analyzed and the particular structure being sought (e.g. a color anatomical image, a general purpose video frame etc.). Hence the statistics collected for the regions will also change.

Once the regions have been classified, the algorithm identifies as boundary regions all those exterior regions that share an edge with an interior region. We construct the Delaunay triangulation and select those edges that connect the seed points in the neighboring boundary regions. This defines an approximation of the outline of the processed structure. In order to improve the accuracy of the results, we add a seed point on the midpoint of each edge of every boundary region, recalculate the Voronoi diagram with these new seeds, and repeat the process. Before adding new seed points, though, the user has the option of manually reclassifying some boundary regions as exterior regions in order to prevent the algorithm from focusing on unwanted details and to minimize the inaccuracies introduced by deficiencies in the classification statistics. In addition, the user may stipulate that the algorithm runs automatically for a fixed number of iterations and then views the results, or 
he/she can choose to interact with the data after each step. A pseudo-code for the algorithm is described in Fig. 3.

Our algorithm benefits, in particular, from its robustness. No limitations are imposed by the design on the nature of the statistics used to classify the Voronoi regions, so the algorithm can accommodate a wide variety of measurements for each region. Flexibility in the specification of classification statistics allows the algorithm to operate on virtually any type of image from full-color anatomical images to video frames. In addition, our algorithm can be easily extended to include considerations such as geometric location in an image in its classification scheme due to the region-based nature of our approach. Such extensions could conceivably improve segmentation results in cases in which the search can be concentrated on only one section of an image.

\subsection{Implementation}

In order to test our segmentation algorithm, we have developed an interactive program to generate outlines of the lungs in full-color slides of cross-sectional slices of the Visible Human male. In designing the program we addressed a variety of important issues, including generating and displaying Voronoi diagrams overlayed on the color slices, developing an efficient method for visiting each region, creating useful classification criteria for identifying the color lung regions, and numerous interface details.

In light of the above discussion, our program must be able to generate and manipulate Voronoi diagrams and Delaunay triangulations for various seed point distributions. We use the qhull, version 2.1, program from the Geometry Center [21], which implements a variant of the Quickhull algorithm to calculate convex hulls, Voronoi diagrams, and Delaunay triangulations.

Our program generates an initial Voronoi diagram for a test image by first allowing the user to choose to identify interactively seed points by clicking on the image with the cursor or to have the program generate some number of random seed points and then running qhull on the seed points distribution. In order to display accurately the resulting Voronoi diagram, including proper representation and display for infinite regions, we have developed a utility which processes the data output by qhull, and renders the Voronoi regions using our own algorithm. Once the initial Voronoi diagram has been generated, the program visits each region to accumulate classification statistics and makes a determination as to the identity of the region.

We determined the experimental statistics for the lung by analyzing a number of sample matrices (around 60) of size $5 \times 5$ pixels, in different color spaces. We looked at different statistics (e.g. average, variance) in the RGB (Red, Green, Blue) and HVC (Hue, Value Chroma) color spaces. The HVC system represents images with hue, value, and chroma (or saturation) levels, which in effect separate the luminant and chromatic constituents of a color [19]. The hue indicates the wavelength of light reflected from or transmitted through an object and is normally indicated with a color name, whereas the value, or brightness, indicates the total amount of light in a color, with a zero value being back. The saturation (chroma) denotes the proximity of the color to monotone with a zero value meaning gray [18]. We found that the lungs could be characterized by a relatively high variance in saturation values and a low variance in the red intensity. Thus, we used these statistics as our classification criteria. Specifically, a region is classified as interior if the variance in saturation in the region is $\geq 21.5$ and the red variance is $\leq 0.01$ or if the saturation variance is $\geq 20.0$ and the red variance is $\leq 0.0015$. Otherwise, the program identifies the region as exterior. Since the image data we used for our experiments were stored in RGB format, we had to include code to perform a transformation from the RGB color space to the HVC color space in order to measure values for saturation.

Our program uses the following equations to convert the original data first to the CIE $L^{*} a^{*} b$ color space, as proposed by Gong and Sakauchi [19], and then to HVC values

$$
\begin{aligned}
& X=0.607 \cdot R+0.174 \cdot G+0.201 \cdot B \\
& Y=0.299 \cdot R+0.587 \cdot G+0.144 \cdot B \\
& Z=0.066 \cdot G+1.117 \cdot B \\
& L^{*}=116 \cdot Y^{1 / 3}-16 \\
& a^{*}=500 \cdot\left(X^{1 / 3}-Y^{1 / 3}\right) \\
& b^{*}=500 \cdot\left(Y^{1 / 3}-Z^{1 / 3}\right) \\
& H=\tan ^{-1}\left(\frac{b^{*}}{a^{*}}\right) \\
& C=L^{*} \\
& V=\sqrt{a^{2}+b^{2}}
\end{aligned}
$$

In order to calculate the variance in saturation and red values in a particular region, we make use of a modified seed fill algorithm [20] to visit all the pixels in a region. We should note that in the original algorithm, by Bertin et al., a minimal enclosing box (rectangle) was chosen instead, which included the Voronoi region. All the pixels in the rectangle were visited, and a search for the nearest seed was done. Since such enclosing boxes for adjacent Voronoi regions have to overlap, some pixels were visited more than once. In our simple and elegant method, we guarantee that each pixel in each Voronoi region is visited exactly once.

The program stores the Voronoi diagram of a particular iteration in memory, and starting at a region seed point, it performs a standard seed fill on the region without rendering the results to the screen. However, in addition to marking 


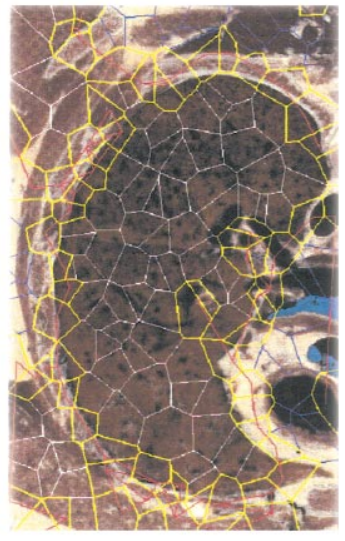

(a)

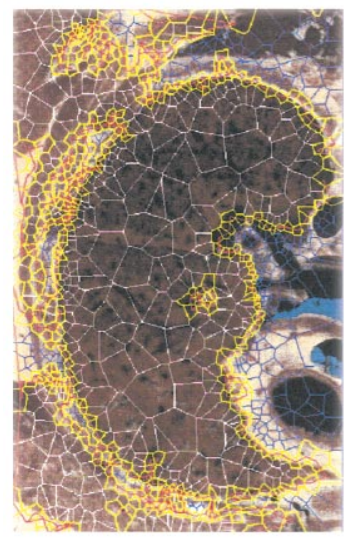

(c)

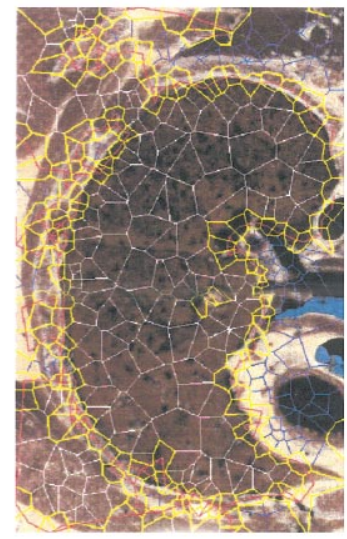

(b)

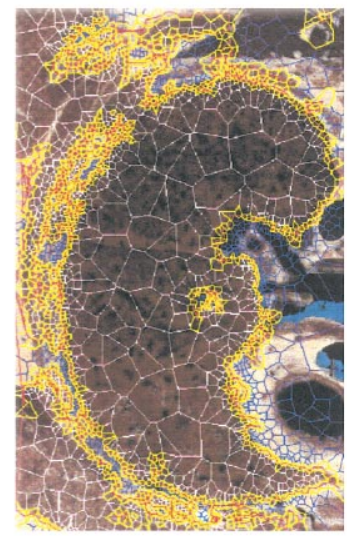

(d)
Fig. 4. Sequence of images for an automatic run on an initial random distribution of 200 seed points: (a) first run; (b) second run; (c) third run; and (d) fourth run.

each pixel it visits, the program also accumulates the red value and saturation level for these pixels. Thus, by the time the fill ends, every pixel has been visited once, and the variance in the two statistics can be calculated.

After visiting a region, the program applies the tests discussed above to classify the region as either interior or exterior. The process repeats for each region in the diagram, and then the program determines which exterior regions are adjacent to interior regions. These adjacent regions are classified as boundary regions. At this point, the user is presented with a display of the image being processed with the current Voronoi diagram superimposed upon it. The regions in the diagram are color coded such that white indicates an interior region, yellow a boundary region, and blue an exterior region. The user can select a menu option to allow for interactive reclassification of boundary regions as discussed in the previous section, or he/she can choose to have the program iterate automatically.

If the user reclassifies any boundary regions, they are removed from the list of boundaries maintained by the program. When the user has finished reclassifying regions, or if the program has been set to iterate automatically, only those regions that appear in the list of boundary regions are subdivided by having seed points planted on their edges. The program feeds this new set of seed points into the algorithm as input for the next iteration. When the user is satisfied with the results of the segmentation, he/she can choose to have a boundary line drawn on the image. The program generates the line by first running qhull to calculate the Delaunay triangulation of all the seed points in the image and then drawing only those segments of the triangulation which join two boundary regions. In testing we found that the resulting line will, in most cases, approximate the outline of the lung quite well but will also contain some loops and dead end off-shoot segments due to the fact that the algorithm normally cannot generate a one-region thick layer of boundary regions around the entire lung. Usually, at least one section of the lung will have a boundary two or more regions thick due to misidentification of an exterior region as interior. As might be expected, this problem is more noticeable in fully automatic runs than in those for which the user has provided some guidance to the algorithm. A single continuous boundary line can quite easily be produced from the triangulation data by simply running a fill on the boundary generated from the triangulation data by simply running a fill on the boundary generated from the triangulation and drawing only those segments that come into contact with the fill color. Another option is to fit a spline boundary to the approximate outline. The user also has some standard choices accessible via pull-down menus, such as loading and saving images and saving boundary data. The save boundary option allows the user to save to a text file the coordinates of and color values at the seed points of the boundary regions used to form the outline of the lung. This data will be accumulated for each slide in the Visible Human data set which contains a section of lung, and it will be used to help generate a 3D voxel-based representation of the lung.

\subsection{Time complexity}

The time complexity of constructing the Voronoi diagram for $|V|$ seed points is $\mathrm{O}(|V| \log |V|)$ and the Delaunay triangulation is obtained in additional $\mathrm{O}(|V|)$ time [21]. In addition, since each pixel in a region is visited a constant number of times, and we visit each region at most once in an iteration, the entire process of visiting and reclassifying takes $\mathrm{O}(N)$ time, where $\mathrm{O}(N)$ is the number of pixels in the image. Thus one iteration of the algorithm has an overall time complexity of $\mathrm{O}(\mathrm{N}+|V| \log |V|)$. Since the number of iterations is a constant, user pre-defined parameter, the time complexity of the algorithm is $\mathrm{O}(\mathrm{N}+|V| \log |V|)$.

\section{Results}

We obtained a number of interesting results while experimenting with our segmentation system. For example, we found that using different sorts of random distributions, 


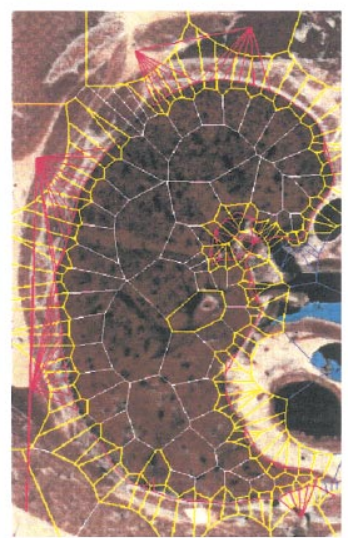

(a)

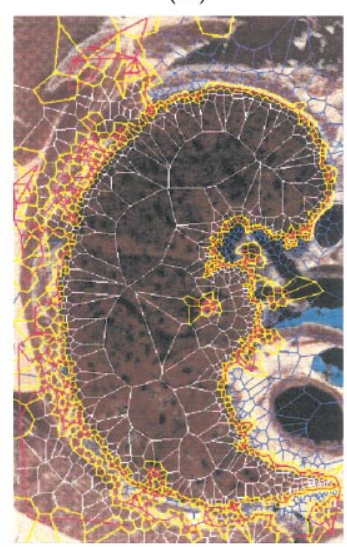

(c)

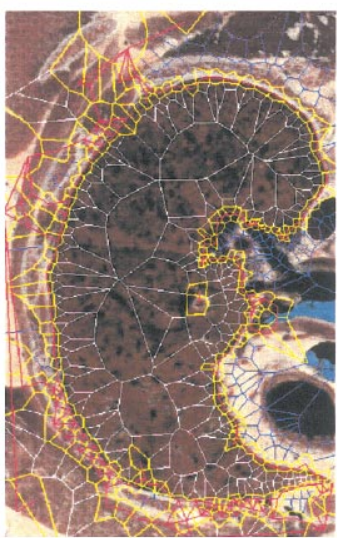

(b)

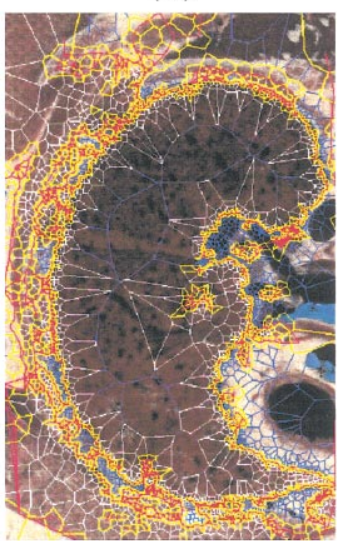

(d)

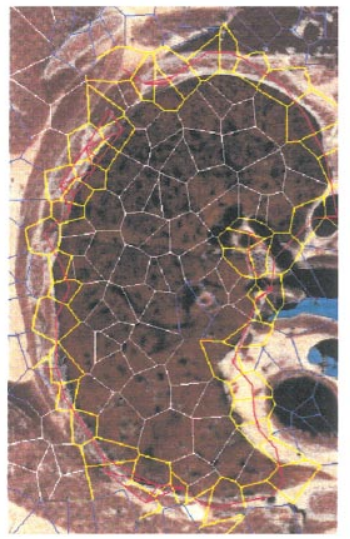

(a)

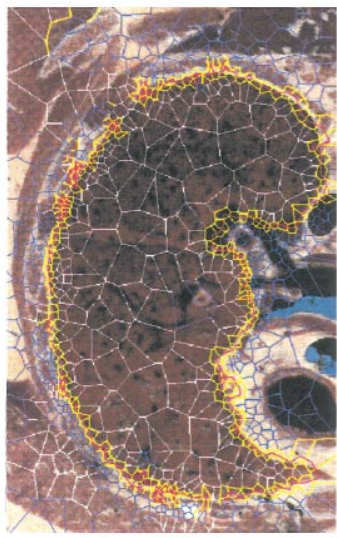

(c)

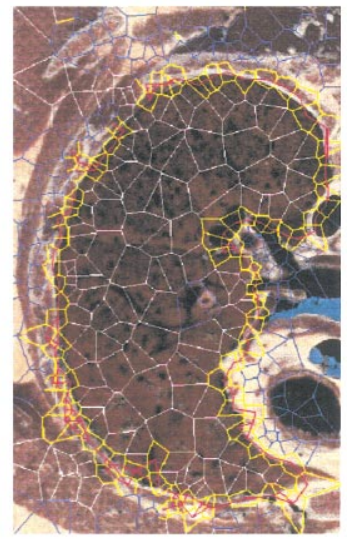

(b)

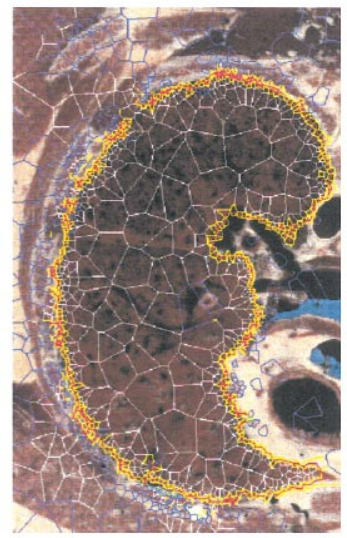

(d)
Fig. 5. Sequence of images for an automatic run on an initial manual distribution of 200 seed points: (a) first run; (b) second run; (c) third run; and (d) fourth run.

such as Poisson and binomial, for the initial seed points make no appreciable difference in the final results. We also noticed that a set of manually distributed seed points produces only a slightly higher quality outline, in the same number of iterations, than will a random distribution of the same number of points. In this case, a "high quality" outline is one which corresponds closely to the outline generated by an experienced anatomical illustrator viewing the same slide. Figs. 4 and 5 show a comparison of the intermediate steps and the final result of running the program in an automatic mode for iterations on random and manual initial distributions, respectively.

In addition, as might be expected, human interaction can greatly improve the results of the segmentation process. As shown in Figs. 6 and 7, the results of four iterations with interactive reclassification for regions after each step tend to display a lower density of regions in difficult to segment areas than do those of a fully automatic run, since the user can force the program to ignore certain areas on which it might otherwise concentrate too much effort. Also, the images are devoid of the excessive noise produced in the fully automatic mode. As mentioned above, having random
Fig. 6. Sequence of images for an interactive run on an initial random distribution of 200 seed points: (a) first run; (b) second run; (c) third run; and (d) fourth run.

or manual seed distributions make no visible difference in the final segmentation of the lung region, so a random distribution, which requires less user interaction, should be used by default.

Clearly, our region-based segmentation technique produces visually accurate results (validated by an experienced anatomist) even without any user interaction. More importantly, though, with only a minimal amount of user input, the program can produce a highly accurate representation of the boundary of a lung. This low requirement for user input becomes particularly important in our case when one considers that the lungs appear in over 300 slices in the Visible Human data set. Using our approach the slices can be processed efficiently and accurately.

\section{Conclusions}

We have proposed a novel and efficient method of color image segmentation using a semi-automated region-based approach, and we have proven its effectiveness by implementing it in a system which generates outlines of lungs in 


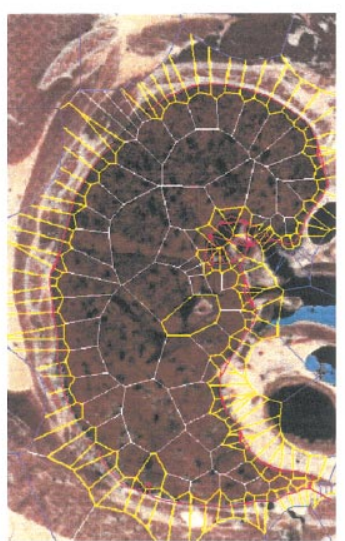

(a)

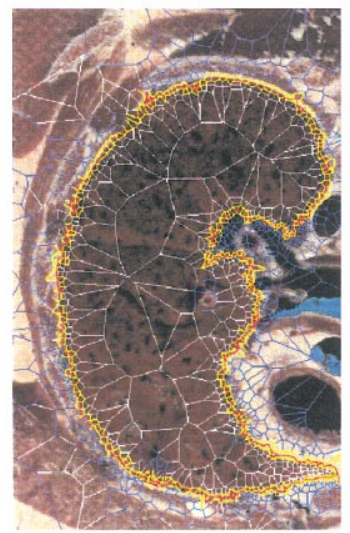

(c)

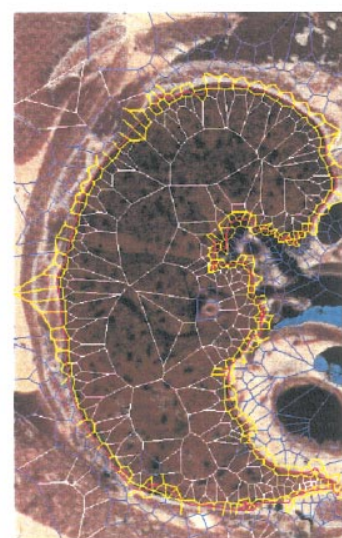

(b)

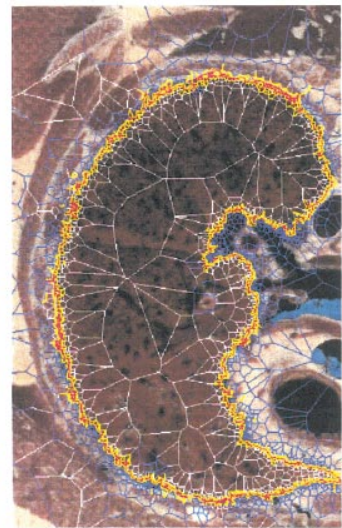

(d)
Fig. 7. Sequence of images for an interactive run on an initial manual distribution of 200 seed points: (a) first run; (b) second run; (c) third run; and (d) fourth run.

slides from the Visible Human Project. This work is a first attempt to attack the complex issue of outlining fine anatomical structures in color medical images. Building on the work of Ref. [16], we extended the original gray scale work to full-color data and developed a variety of improvements and modifications, chief among them being the inclusion of user interaction and efficient region processing.

Our experimental results, including determination of an experimental classifier for the lungs, indicate the efficacy of our method and point the way toward a number of future developments. More experiments must be carried out to determine classification statistics for a wider variety of anatomical structures so that a more rigorous mathematical description of such structures can be developed. In this regard, our experiments lead us to believe that saturation values will play an important part in distinguishing between structures. Future work will also focus on adapting recent work in texture segmentation to our classification scheme. In addition, as mentioned earlier, our approach allows for easy extension of classification criteria to include measures of geometric location and other statistics which should be implemented and tested on the anatomical data.

The output from our application has already been used as a foundation for creating 3D representations of the lungs. After further refinement, the program will play an important role in providing data for a 3D anatomical imaging system designed to use the Visible Human Project data to improve upon current techniques in anatomy education. Extension of this method to a 3D region-based color segmentation is possible, if we employ the 3D Voronoi diagram to process the input data in a color voxel representation.

\section{Acknowledgements}

We would like to thank Swaroop Hosakere and Asim Khan for participating in the implementation of the algorithm.

\section{References}

[1] Ackerman MJ. Fact sheet: the visible human project. National Library of Medicine, 1995

[2] Spitzer V, et al. The visible human male: a technical report. Journal of the American Medical Informatics Association 1995.

[3] Bean C, Imielińska C, Molholt P, Laino-Pepper L. Symbolic and spatial knowledge model. Proceedings of the First User Conference of the National Library of Medicine's Visible Human Project, 8-9 October 1996, Bethesda, MD 1996.

[4] http://cpmcnet.columbia.edu/vesalius.

[5] Imielinska C, Molholt P, Bean C, Laino-Pepper L, Morris D. Network-based medical visualization. Technical Report No. 9604, Sevens Institute of Technology, April 1996.

[6] Imielinska C, Molholt P, Bean C, Laino-Pepper L, Soliz E, Thumann R. The VESALIUS project: visualization of lungs and bronchial tree anatomy lesson. Proceedings of the First User Conference of the National Library of Medicine's Visible Human Project, 8-9 October 1996, Bethesda, MD 1996.

[7] Molholt P, Bean C, Imielinska C, Laino-Pepper L. The VESALIUS project: creating a network-based anatomy curriculum. Technical Report No. 9605, Stevens Institute of Technology, April 1996.

[8] Wacholder N, Imielinska C, Johnson S, Soliz E, Molholt P. Implications of 3D visualization for medical education. Proceedings of the Medicine Meets Virtual Reality:6 (MMVR:6) Conference, San Diego, California, January 1998.

[9] Wacholder N, Imielinska C, Klavans J, Molholt P, Soliz E. Semantic relations in a medical digital libraries. Proceedings of the American Digital Library Conference, Santa Barbara, April 1998.

[10] Ronfard R. Region-based strategies for active contour models. International Journal of Computer Vision 1985;13(2):229-51.

[11] Kass M, Witkin A, Terzopoulos D. Snakes: active contour models. International Journal of Computer Vision 1988;1(4):321-31.

[12] Jones TN, Metaxas DN. Segmentation using deformable models with affinity-based localization. Proceedings of the First International Joint Conference for CVRMedMRCAS, Grenoble, 1997.

[13] Celenk M, Smith SH. Gross segmentation of color images of natural scenes for computer vision systems. Applications of Artificial Intelligence 1986;3:333-44.

[14] Liu J, Yang Y. Multiresolution color image segmentation. IEEE Transactions on Pattern Analysis and Machine Intelligence 1994;16(7):689-700.

[15] Ohta YI, Kanade T, Sakai T. Color information for region segmentation. Computer Graphics and Image Processing 1980;13(5):222-41.

[16] Bertin E., Parazza F., Chassery J.M. Segmentation and measurement 
based on 3D Voronoi diagram: application to confocal microscopy. Computerized Medical Imaging and Graphics 17(2):175-82.

[17] Preparata FP, Shamos MI. Computational geometry: an introduction. New York: Springer, 1985.

[18] Adobe Photoshop User's Manual, Adobe Inc., 1994.

[19] Gong Y, Sakauchi M. Detection of regions matching specified chromatic features. Computer Vision and Image Understanding 1995;61(2):263-9.

[20] Foley JD, van Dam A, Feiner SK, Hughes JF. Computer graphics: principles and practice. 2 ed.. Reading, MA: Addison-Wesley, 1990.

[21] Barber CB, Dobkin DP, Huhdanpaa H. The quickhull algorithm for convex hull. The Geometry Center, University of Minnesota, 1995.

Celina Imielińska is an Associate Research Scientist at the Columbia University College of Physicians and Surgeons, Office of Scholarly Resources, and the Department of Computer Science. She is one of the founders of the Vesalius ${ }^{\text {TM }}$ Project $^{1}$, an interdisciplinary effort at Columbia University to create a network-based, platform-independent electronic "course" in anatomy. Shee took the lead on the technical problems of the medical image segmentation and 3D medical visualization. Dr. Imielińska, has been trained as computer scientist and electrical engineer. She holds BE, ME in Electrical Engineering from the Technical University of Gdánsk, in Poland, and $\mathrm{MS}$ and $\mathrm{PhD}$ in Computer Science from Rutgers University. Her current interests are in 3D visualization, computer graphics, computational geometry, image processing and approximate algorithms for optimization problems. While writing her $\mathrm{PhD}$ thesis on approximation algorithms for Euclidean combinatorial problems, she worked for two years as a research associate at the General Robotics and Active Sensory Perception Laboratory at the University of Pennsylvania on vision and robotics problems. After joining faculty at the Electrical Engineering and Computer Science Department at Stevens Institute of Technology, she had developed computer graphics program. She joined Columbia University in 1998 as an Associate Research Scientist at the Department of Computer Science and the Office of Scholarly Resources of the College of Physicians and Surgeons of Columbia University. She has been supported by the NSF POWRE grant on "Technical Challenges of 3D Visualization of Large Color Data Sets". She is the leader of the Columbia team on the NLM-funded contract on "Visible Human Project registration and Segmentation Toolkit", where she is developing hybrid segmentation methods for medical images. In addition to conducting research and developing methodology for image processing and 3D modeling, she has been involved in developing the actual 3D visualizations of anatomy from the Visible Human dataset, which have been used in electronic anatomy curriculum at Columbia's medical school.
Michael S. Downes is a PhD Student in the Computer Science Division of the Electrical Engineering and Computer Sciences Department at the University of California, Berkeley. His research interests include scientific visualization, physically-based modeling, and virtual environments for simulation and training. He earned his BE in computer engineering from the Stevens Institute of Technology in 1996.

Wei Yuan received MS degree from Stanford University Department of Computer Science. He has worked on multiple research projects in databases and electronic commerce. With two years of experience in developing database and Internet applications, he has a strong interest in distributed systems and computer graphics. His specialties includes Java programming, computer graphics and distributed systems design. $\mathrm{He}$ earned his $\mathrm{BE}$ in computer engineering from Stevens Institute of Technology in 1996

\footnotetext{
${ }^{1}$ Trademark held by Columbia University. The Vesalius ${ }^{\text {Tu }}$ Project is named after Andreas Vesalius, a sixteenth century anatomist whose work laid the foundations for all subsequent anatomical research. The Columbia University Health Sciences Library own several first editions of his work.
} 